\title{
Correction to: What is the predictor of the intraoperative body temperature in abdominal surgery?
}

\author{
Ryohei Miyazaki $^{1}$ [D $\cdot$ Sumio Hoka ${ }^{2}$
}

Published online: 20 December 2018

C Japanese Society of Anesthesiologists 2018

\section{Correction to: Journal of Anesthesia} https://doi.org/10.1007/s00540-018-2585-6

In the original publication of the article, Table 1 was incorrectly published.

The correct Table 1 is

The original article can be found online at https://doi.org/10.1007/ s00540-018-2585-6.

Ryohei Miyazaki

miyaryou@kuaccm.med.kyushu-u.ac.jp

1 Operating Rooms, Kyushu University Hospital, 3-1-1 Maidashi, Higashi-ku, Fukuoka 812-8582, Japan

2 Department of Anesthesiology and Critical Care Medicine, Graduate School of Medical Sciences, Kyushu University, Fukuoka, Japan
Table 1 Overall patients' demographic and clinical characteristics

\begin{tabular}{ll}
\hline Characteristic & $n=2574$ \\
\hline Age (years) & $64 \pm 14$ \\
Height $(\mathrm{m})$ & $1.61 \pm 0.09$ \\
Weight $(\mathrm{kg})$ & $59.4 \pm 11.8$ \\
BMI $\left(\mathrm{kg} / \mathrm{m}^{2}\right)$ & $22.7 \pm 3.5$ \\
Sex, $n(\%)$ & \\
Female & $977(38 \%)$ \\
Male & $1597(62 \%)$ \\
ASAPS, $n(\%)$ & \\
Category 1 & $393(15 \%)$ \\
Category 2 & $1689(66 \%)$ \\
Category 3 & $492(19 \%)$ \\
Surgery type, $n(\%)$ & \\
Laparoscopic & $969(38 \%)$ \\
Open & $1605(62 \%)$ \\
Time to incision $(\mathrm{min})$ & $50 \pm 16$ \\
Operative time (min) & $270 \pm 152$ \\
Epidural anesthesia, $n(\%)$ & $1415(55 \%)$ \\
Initial body temperature $\left({ }^{\circ} \mathrm{C}\right)$ & $36.6 \pm 0.5$ \\
Blanket type, $n(\%)$ & \\
Overbody & $1520(59 \%)$ \\
Underbody & $1054(41 \%)$ \\
Blood loss $(\mathrm{g})$ & $301 \pm 477$ \\
Urine output $(\mathrm{ml})$ & $817 \pm 620$ \\
\hline
\end{tabular}

Time to incision: time between anesthetic induction and surgical incision. Initial body temperature: temperature at the time of the surgical incision. Data are presented as number $(\%)$ or mean \pm standard deviation

$B M I$ body mass index, ASAPS American Society of Anesthesiologists physical status score

Publisher's Note Springer Nature remains neutral with regard to jurisdictional claims in published maps and institutional affiliations. 